\title{
分子动力学方法研究聚甲基乙烯基醚/水体系中 氢键和准氢键的结构与分布
}

\author{
吴荣亮，吉青，孔滨，杨小震*
}

北京分子科学国家实验室，高分子物理与化学国家重点实验室，中国科学院化学研究所，北京 100080

* 联系人, E-mail: yangx@iccas.ac.cn

收稿日期: 2008-03-06; 接受日期: 2008-03-16

国家自然科学基金(批准号: 20474073, 20490220, 20674090 和 90612015)和国家重大基础研究发展计划(批准号: G1999064800)资助项目

摘要用分子动力学方法模拟室温下不同浓度的聚甲基乙烯基醚/水体系的微观 溶剂化结构. 得到的径向分布函数和氢键给体和受体距离分布表明, 聚合物与水形 成的氢键比水之间形成的氢键短约 $0.005 \mathrm{~nm}$. 准氢键 $\mathrm{C}-\mathrm{H} \cdots \mathrm{O}$ 的数目是范德华作用 对的 $7.2 \%$. 我们发现, 在各浓度下, 水分子并不能均匀地分布在聚合物结构单元上, 即使在很稀的溶液 $(3.3 \%$, 质量分数) 中, 仍然有 $10 \%$ 左右的醚氧没有和水分子形成 氢键. 这说明在溶液中, 不但高分子链间有紧密的接触, 而且高分子链内的链段间 也有紧密的接触, 导致链上的一些醚氧不能和水分子有效地接触而形成氢键. 准氢 键随浓度的变化和氢键的变化趋势类似, 但形成准氢键的结构单元数目与形成氢键 的结构单元数目比值在 0.2 附近. 文献上用动态 DSC 测量低分子量聚甲基乙烯基梄 (PVME)水溶液的相转变焓发现, 在浓度为 $30 \%$ 左右有一转折, 与本模拟所得出的在 浓度为 $27 \%$ 左右氢键和准氢键比例的转折相关, 这给相转变焓的转折点提供了分子 尺度的微观解释. 另外, 浓度小于 $54 \%$ 的溶液中存在 “自由水”, 在 $86 \%$ 的浓溶液中每 个结构单元大约与 1.56 个水分子缔合.

\section{关键词}

聚甲基乙烯基醚(PVME)

分子动力学

分子模拟

氢键

准氢键

\section{1 研究背景}

很多水溶性的高分子溶液体系都具有低临界共 溶温度 (LCST), 如聚氧乙烯 $(\mathrm{PEO})^{[1]}$ 、聚乙烯醇 $(\mathrm{PVA})^{[2]}$ 和聚甲基乙烯基醚(PVME) ${ }^{[2 \sim 5]}$ 等, 它们在人 造肌肉 ${ }^{[6]}$ 、药物载体 ${ }^{[7]}$ 和分子识别 ${ }^{[8]}$ 等领域具有其它 聚合物无法比拟的应用前景.

PVME $/ \mathrm{H}_{2} \mathrm{O}$ 体系的LCST曲线具有两个极小值, 呈“W”形 ${ }^{[9]}$, 且接近室温 $\left(37^{\circ} \mathrm{C}\right)^{[4]}$. PVME和水之间特 殊的相互作用被认为是产生这种“ $\mathrm{W}$ ”形相图的重要 原因 $[10]$. 该体系的分子间相互作用包括亲水相互作
用和亲油相互作用. 亲水相互作用即醚氧和水之间 形成的氢键; 而亲油相互作用则主要体现在与醚氧 相连的甲基上, 它可以改变聚合物分子链周围正常 的水分子氢键网络结构而形成特殊的分子团簇, Némethy 和Scheraga ${ }^{[11]}$ 以及 Frank和Evans ${ }^{[12]}$ 称之为“冰 山”(icebergs).

对于上述现象，实验上已有广泛的研究 ${ }^{[2,4,5,10]}$, 主要方法有近红外、黏度法和量热等。通过测量 $\mathrm{PVME} / \mathrm{H}_{2} \mathrm{O}$ 体系的红外谱带位移、特征温度和相变吸 热等，人们推测该体系中的水分子可以分为 3 类 ${ }^{[10]}$ : 平均 2.7 个水分子和PVME的醚氧形成氢键; 而另有 
2.3 个水分子与每个结构单元的亲油基团形成“冰山” 结构; 其余有超过 5 个水分子(每结构单元)为“自由 水”, 即不与聚合物形成特定的结构. 为了更详细地 了解PVME和水分子之间的相互作用及其可能的团 簇结构, 本组前期工作 ${ }^{[5]}$ 利用量子力学 MP2 方法和 $6-31+\mathrm{G}^{*}$ 基组，通过优化PVME的模型化合物二甲醚 (DME)(其中一个甲基被氛代)和 2 5 个水分子形成的 团簇，得到了不同数目的水分子和DME结合的各种 构型及其相对能量. 发现 $\mathrm{DME}$ 的 $\mathrm{CH}_{3}$ 基团和水确实 存在特殊的相互作用, 这种相互作用介于氢键和范 德华相互作用之间, 我们称之为“准氢键”[5]. 并且该 工作认为在较低的PVME浓度下, 致使 $v(\mathrm{C}-\mathrm{H})$ 红外 谱带发生蓝移的主要因素是准氢键, 而在高浓度区 (40\%)起主导作用的是醚氧和水之间形成的氢键. 并 在此基础上建立了 PVME和水相互作用的统计模型. 发现由模型得到的红外谱带位移峰值位置与实验结 果在 $60 \%$ 100\%范围内吻合得很好, 并推断当PVME 浓度达到 69\%(平均 $1.5 \mathrm{~W} / \mathrm{M}$ ) 时, 分子链中所有的结 构单元都已与水直接或间接地缔合成氢键, 并且在 这个浓度区间内水分子和PVME分子链结构单元的 氢键缔合并不是均一的. 虽然通过量子力学计算可 以得到PVME结构单元和水缔合的具体结构, 所提出 的模型在其相应浓度范围内也可以和实验很好地吻 合, 但是该研究依据的模型化合物体系缺少高分子 链拓扑结构的影响和动力学特征.

原子尺度的分子动力学模拟在研究这类问题时 具有其独特的优势, 它能够细致地描述各种系综下 体系的统计性质及动力学行为. 为此, 我们利用合理 的经验势函数力场对不同浓度下的 $\mathrm{PVME} / \mathrm{H}_{2} \mathrm{O}$ 体系 进行了全原子分子动力学模拟. 分析了不同浓度下 PVME 和水形成的氢键和准氢键的结构及分布, 以及 在聚合物结构单元上分布的特征.

\section{2 理论方法}

模拟所用 PVME 分子链均为含有 44 个主链碳的 无规立构聚合物, 所有结构单元均头-尾相连. 聚合 物链的最可几立构序列通过 Monte Carlo 方法以立构 比即 meso: racemic $=1: 1$ 生成. 具体做法是: 首先通 过 Monte Carlo 方法生成 1000 个给定立构比的模板链 序列, 每个模板链序列含 10000 个结构单元. 统计不
同长度立构序列的比例(最多计算到含 4 个结构单元 长度的立构序列). 然后, 用同样的方法生成含 44 个 结构单元的链序列, 样本数仍为 1000 , 立构比与模板 链相同. 将这些短链样本立构序列的比例与模板链 进行对比，以方差最小的原则篮选出与模板链立构 序列分布最接近的样本，即该长度下分子链的最可 几立构序列.

表 1 列出了所模拟的各体系中所含PVME分子链 数 $n_{\mathrm{p}}$, 水分子数 $n_{\mathrm{w}}$, 水分子数与聚合物结构单元数之 比 $n_{\mathrm{w}} / n_{\text {mon }}$ 以及聚合物的重量浓度 $c_{\mathrm{p}}$. 多链模型由材料 模拟软件Materials Studio 3.1 的Amorphous Builder模 块构造, 其中母链使用最可几立构序列. 每个体系构 造 20 个随机构型, 从中选择能量最低者作为模拟的 起始结构. 能量优化数百步后用 Gromacs $3.3^{[13]}$ 的 “genbox”命令将高分子体系“溶解”于水中. 所有的 MD模拟过程也均在Gromacs下完成.

表 1 模拟体系的具体组分

\begin{tabular}{rrcr}
\hline$n_{\mathrm{p}}$ & $n_{\mathrm{w}}$ & $n_{\mathrm{w}} / n_{\text {mon }}$ & $c_{\mathrm{p}}(\%)$ \\
\hline 0 & 2218 & $/$ & 0.0 \\
1 & 2133 & 96.95 & 3.3 \\
4 & 1905 & 21.65 & 13.0 \\
8 & 1585 & 9.02 & 26.4 \\
12 & 1280 & 4.85 & 40.0 \\
16 & 987 & 2.80 & 53.5 \\
24 & 714 & 1.35 & 70.5 \\
30 & 346 & 0.52 & 86.0 \\
40 & 0 & 0.00 & 100.0 \\
\hline
\end{tabular}

每个溶液体系经共轭梯度法能量优化数百步后 进行恒粒子数-恒压-恒温(NPT)分子动力学模拟 $10 \mathrm{ns,}$ 然后进行正则系综(NVT)模拟 $20 \mathrm{~ns}$. 纯水及溶液体 系温度均控制在 $300 \mathrm{~K}$. 纯PVME体系在高温 $(600 \mathrm{~K})$ 平衡后降温至 $300 \mathrm{~K}$, 然后继续NPT模拟 $10 \mathrm{~ns}$. 温度 耦合采用 Nose-Hoover ${ }^{[14,15]}$ 方法，压力耦合采用 Parrinello-Rahman ${ }^{[16]}$ 方法，非键作用截断半径为 1.2 $\mathrm{nm}$, 使用Particle Mesh Ewald (PME) ${ }^{[17,18]}$ 进行长程静 电作用较正, 在模拟过程中, 高分子的键长通过 Lincs ${ }^{[19]}$ 方法进行限定，水分子的结构由Settle ${ }^{[20]}$ 方法 限定，时间步长 $1 \mathrm{fs}$, 每 $1 \mathrm{ps}$ 记录一次轨迹.

经验势函数采用方程(1)的形式，其中前两项分 别为键长和键角的简谐势, 第三项为二面角势函数, 后两项是非键相互作用，包括Lennard-Jones (L-J 12-6) 
势和静电作用势．选用TIP4P水模型 ${ }^{[21,22]}$ 和OPLS全原 子力场 ${ }^{[23,24]}$. 表 2 给出了各原子的非键作用参数, 其 中 $\varepsilon$ 和 $\sigma$ 为L-J 势的势阱和碰撞直径, $q$ 为各原子的极化 电荷, $m$ 为原子质量. 不同原子之间的非键作用参数采 用几何平均的方法, 聚合物分子链中相隔 3 个键以上 的原子之间看作普通的非键相互作用，而 1-4 相互作 用的非键作用能均为普通非键作用能的 $1 / 2$. PVME的 OPLS力场参数和TIP4P水模型参数都可以在Gromacs 中得到.

$$
\begin{aligned}
V= & \frac{1}{2} k_{b}\left(l-l_{0}\right)^{2}+\frac{1}{2} k_{\theta}\left(\theta-\theta_{0}\right)^{2}+\sum_{n=0}^{5} C_{n} \cos ^{n}(\varphi) \\
& +4 \varepsilon\left[\left(\frac{\sigma}{r_{i j}}\right)^{12}-\left(\frac{\sigma}{r_{i j}}\right)^{6}\right]+\frac{q_{i} q_{j}}{4 \pi \varepsilon_{0} r_{i j}} .
\end{aligned}
$$

\section{表 2 OPLS 非键作用参数}

\begin{tabular}{ccccc}
\hline atom & $\varepsilon /{\mathrm{kJ} \cdot \mathrm{mol}^{-1}} \sigma / \mathrm{nm}$ & $q$ & $m$ \\
\hline $\mathrm{PVME}$ & & & & \\
$\mathrm{C}\left(\mathrm{H}_{3}\right)^{\mathrm{a})}$ & 0.276 & 0.350 & -0.18 & 12.0110 \\
$\mathrm{C}\left(\mathrm{H}_{2}\right)^{\mathrm{a})}$ & 0.276 & 0.350 & 0.14 & 12.0110 \\
$\mathrm{C}(\mathrm{H})$ & 0.276 & 0.350 & 0.17 & 12.0110 \\
$\mathrm{C}\left(\mathrm{H}_{3}\right)$ & 0.276 & 0.350 & 0.11 & 12.0110 \\
$\mathrm{C}\left(\mathrm{H}_{2}\right)$ & 0.276 & 0.350 & -0.12 & 12.0110 \\
$\mathrm{O}$ & 0.586 & 0.290 & -0.4 & 15.9994 \\
$\mathrm{H}$ & 0.126 & 0.250 & 0.06 & 1.0080 \\
$\mathrm{TIP} 4 \mathrm{P}$ & & & $0.03^{\mathrm{b})}$ & \\
$\mathrm{OW}$ & 0.649 & 0.315 & 0.00 & 15.9994 \\
$\mathrm{HW}$ & 0.000 & 0.000 & 0.52 & 1.0080 \\
$\mathrm{MW}$ & 0.000 & 0.000 & -1.04 & 0.0000 \\
\hline
\end{tabular}

a) 末端碳原子; b) 和醚氧紧邻的 C 上的 $\mathrm{H} ; \mathrm{c}$ ) TIP4P 水模型中的 亚原子

\section{3 结果与讨论}

\section{1 体系密度随浓度的变化}

体系密度是常用作判断所模拟体系准确程度的 宏观物理量, NPT过程所得到的各体系密度示于图 1. 其中纯水密度 $\left(992.95 \pm 5.69 \mathrm{~kg} / \mathrm{m}^{3}\right)$ 和实验值 $(996.54$ $\mathrm{kg} / \mathrm{m}^{3}$ )吻合很好，各个浓度的PVME水溶液体系的密 度均大于纯水的密度，并且密度在 $70 \%$ 左右有一峰 值, 这和文献报道 ${ }^{[25]}$ 结果一致. PVME本体在 $300 \mathrm{~K}$ 下的密度为 $1019.04 \pm 4.44 \mathrm{~kg} / \mathrm{m}^{3}$, 略小于实验值 ${ }^{[26]}$ $1060.75 \mathrm{~kg} / \mathrm{m}^{3}$, 这是由于所模拟的分子链较短, 故而 密度稍低。

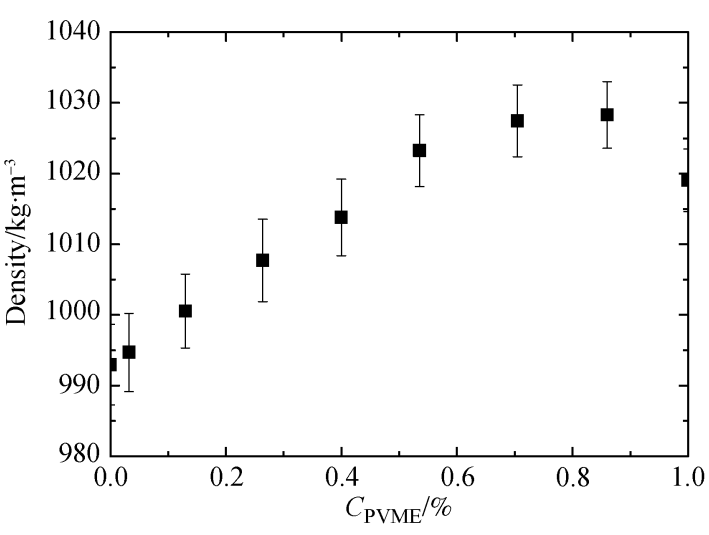

图 1 各浓度下体系的密度

\section{2 氢键及准氢键的作用范围}

PVME水溶液的物理特性主要由聚合物结构单 元和水分子之间的特殊非键相互作用引起，它包括 水分子和聚合物之间的氢键以及由 $\mathrm{C}-\mathrm{H} \cdots \mathrm{O}$ 形成的 准氢键 ${ }^{[5,27]}$. 对于液态体系内粒子间的微观结构和相 互作用直径，通常用径向分布函数(RDF)来表征. 如 $\mathrm{A}$ 和 $\mathrm{B}$ 粒子间的 RDF, $g_{\mathrm{AB}}(r)$, 表示在某个粒子 $\mathrm{A}$ 周围 $r$ $+\mathrm{d} r$ 范围内能够找到粒子 $\mathrm{B}$ 的几率密度.

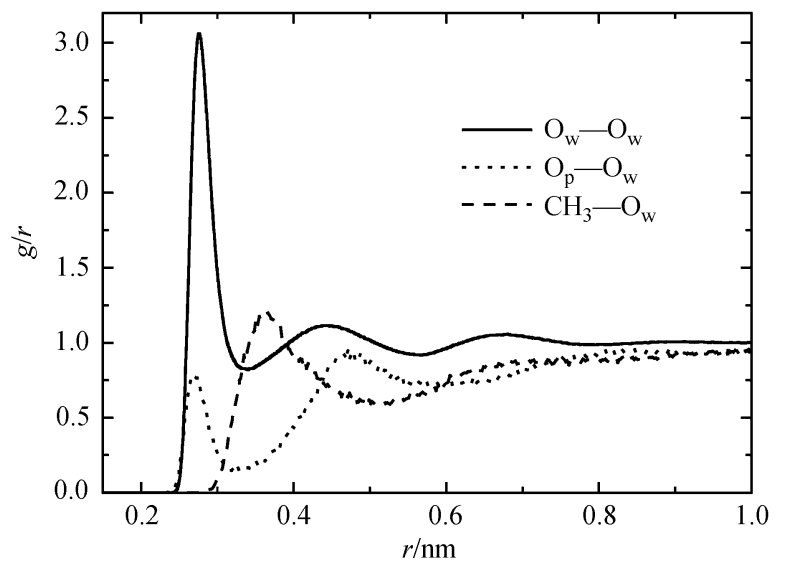

图 2 各原子对的径向分布函数

图 2 给出了 $\mathrm{PVME} / \mathrm{H}_{2} \mathrm{O}$ 体系中水分子的氧原子 之间 $\left(\mathrm{O}_{\mathrm{w}}-\mathrm{O}_{\mathrm{w}}\right) 、 P V M E$ 亲水基团(醚氧)和水分子的氧 原子 $\left(\mathrm{O}_{\mathrm{p}}-\mathrm{O}_{\mathrm{w}}\right)$ 以及侧基上的亲油基团 $\left(\mathrm{CH}_{3}\right)$ 和水分子 的氧原子 $\left(\mathrm{CH}_{3}-\mathrm{O}_{\mathrm{w}}\right)$ 的 RDF. 其中 $\mathrm{O}_{\mathrm{w}}-\mathrm{O}_{\mathrm{w}}$ 和 $\mathrm{O}_{\mathrm{p}}-\mathrm{O}_{\mathrm{w}}$ 的第一近邻主要表示氢键相互作用, 峰值分别为 $0.277 \mathrm{~nm}$ 和 $0.273 \mathrm{~nm}$, 说明 $\mathrm{O}_{\mathrm{p}}-\mathrm{O}_{\mathrm{w}}$ 氢键作用直径稍 短. 另外, $\mathrm{CH}_{3}$ 和 $\mathrm{O}_{\mathrm{w}}$ 的 $\mathrm{RDF}$ 峰值在 $0.367 \mathrm{~nm}$, 由于 
Van der Waals 相互作用对 $\mathrm{CH}_{3}-\mathrm{O}_{\mathrm{w}}$ 原子对的 RDF 有 很大贡献, 所以在这里还不能判定准氢键的准确作 用直径.

\section{3 氢键和准氢键的结构分布}

\subsection{1 氢键的定义及结构分布}

氢键 $(\mathrm{HB})$ 的产生需要电负性的质子供体和拥有 孤对电子的电负性的受体. 在 $P V M E / \mathrm{H}_{2} \mathrm{O}$ 体系中, 醚 氧和水分子的氧原子作为受体相互竞争, 而水分子 则为质子供体. 正是由于这种竞争使得水分子在醚 氧周围形成不同的氢键聚集体 ${ }^{[5]}$.

图 3 展示了 $\mathrm{O}_{\mathrm{w}}-\mathrm{O}_{\mathrm{w}}$ 和 $\mathrm{O}_{\mathrm{p}}-\mathrm{O}_{\mathrm{w}}$ 氢键的键长 (a) 和键 角(b)分布. 其中, 氢键的定义采用几何限制条件 [28], 要求供体 $(\mathrm{D})$ 和受体 $(\mathrm{A})$ 之间的距离 $r(\mathrm{D}-\mathrm{A})<0.35 \mathrm{~nm}$ 以及HDA形成的夹角小于 $30^{\circ}$. 从图中可以发现 $\mathrm{O}_{\mathrm{w}}$ $-\mathrm{O}_{\mathrm{w}}$ 氢键较 $\mathrm{O}_{\mathrm{p}}-\mathrm{O}_{\mathrm{w}}$ 氢键键角分布稍宽, 键长分别为 $0.276 \mathrm{~nm}$ 和 $0.271 \mathrm{~nm}, \mathrm{O}_{\mathrm{p}}-\mathrm{O}_{\mathrm{w}}$ 氢键比 $\mathrm{O}_{\mathrm{w}}-\mathrm{O}_{\mathrm{w}}$ 氢键短 $0.005 \mathrm{~nm}$, 这与量子力学计算结果 ${ }^{[5]}$ 和实验报道结果 [29,30]一致。
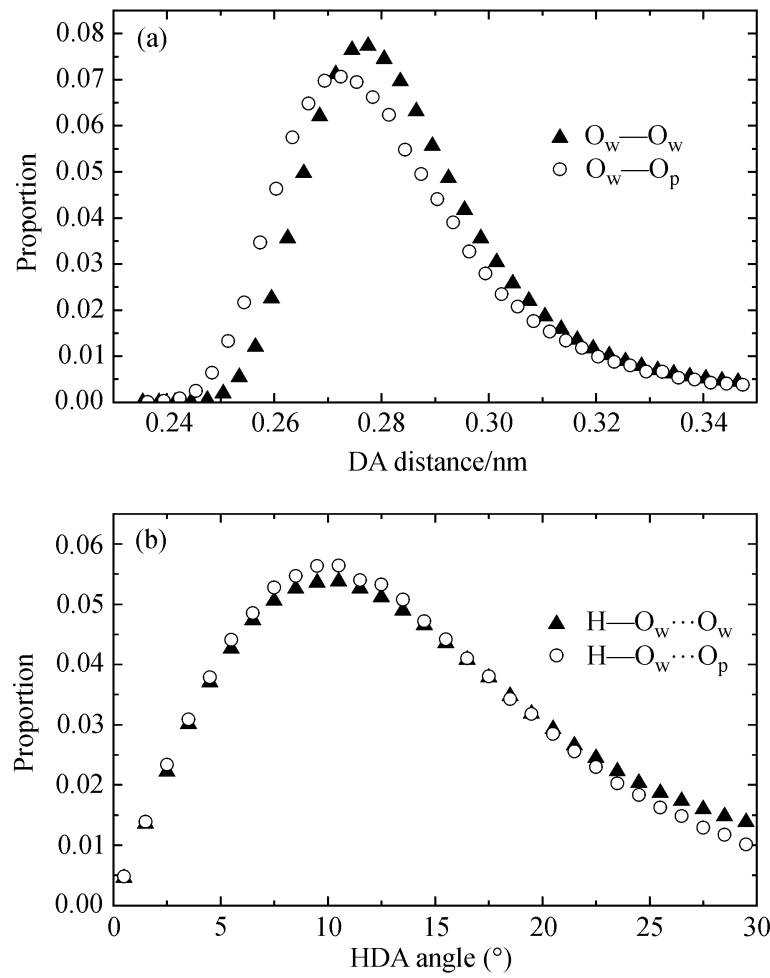

图 3 水分子之间(实三角表示)以及水分子和醚氧之间(空心 圆表示)所形成氢键的 DA 距离分布(a)和 HDA 角的分布(b)

\subsection{2 准氢键的定义与结构分布}

不同的研究小组 ${ }^{[5,27,31,32]}$ 对准氢键的判定标准都 不尽相同. 本工作采用的标准要求 $r(\mathrm{C}-\mathrm{O})<0.372$ $\mathrm{nm}$ 并且 $\mathrm{C}-\mathrm{H} \cdots \mathrm{O}>150^{\circ}$. 其中 $0.372 \mathrm{~nm}$ 为 $\mathrm{C}-\mathrm{O}$ 之间 的范德华直径, $150^{\circ}$ 为量子力学计算 ${ }^{[5]}$ 及 Scheiner ${ }^{[27]}$ 的 结果. 为了区分范德华作用和准氢键作用, 图 4 给出 了准氢键截断直径内所有范德华作用(包括准氢键, 空心圈表示)和准氢键的 $\mathrm{C}-\mathrm{O}$ 距离分布(空心三角表 示). 实三角表示归一化的准氢键 $\mathrm{C}-\mathrm{O}$ 距离分布. 准 氢键 $\mathrm{C}$ - $\mathrm{O}$ 距离峰值在 $0.363 \mathrm{~nm}$ 附近, 比 $\mathrm{RDF}$ 的第一 近邻峰值小 $0.004 \mathrm{~nm}$. 从两条空心点的积分面积来看, 准氢键数目仅占范德华作用对的 $7.2 \%$.

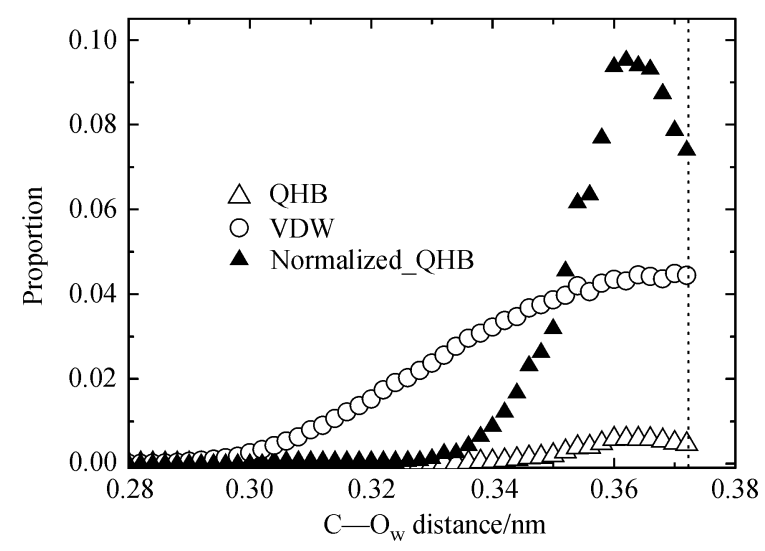

图 4 准氢键 $(\mathrm{QHB})$ 和范德华 $(\mathrm{VDW})$ 作用的 $\mathrm{C}-\mathrm{O}$ 距离分布 实三角表示归一化的准氢键 $\mathrm{C}-\mathrm{O}$ 距离分布. 竖线表示 $\mathrm{C}-\mathrm{O}$ 的 范德华作用直径

\section{4 氢键和准氢键在聚合物结构单元上的分布}

\subsection{1 氢键在聚合物结构单元上的分布}

由于是溶液，水分子和聚合物结构单元应当均 匀分布在体系中形成氢键. 这是小分子体系的概念, 在高分子溶液体系中我们的研究给出了不同的结果. 图 5 表示醚氧和 $i(i=0,1,2)$ 个水分子形成氢键的百分 数. 从图中可以发现, 水分子并不能均匀地分布在聚 合物结构单元上. 即使在最低浓度的溶液 $(3.3 \%)$ 中, 仍然有 $10 \%$ 左右的醚氧没有和水分子形成氢键. 该 结果支持了我们早期的工作 ${ }^{[5]}$ 并说明，在高浓度时， 分子链间有紧密的接触, 阻碍了水分子的介入; 在很 低浓度时，分子链内的链段间也有紧密的接触，仍然 
造成链上的一些单元碰不到水.

图 5 表明, 氢键的浓度在 $27 \%$ 左右有一转折, 转 折点前后氢键浓度的变化率不同. 斜率小的对应于 水的介入缓慢，而斜率大的对应于水的介入迅速. 该 结果与 Durme $\mathrm{e}^{[4]}$ 等人利用动态 DSC 测量低分子量的 PVME水溶液的分相焓的结果相关联。他们发现在 $30 \%$ 左右相转变焓有一拐点. 而我们的发现描述的是 相互作用方式的变化，可以成为该观测的一个注解.

另外, 和同一个醚氧发生两个氢键作用的曲线 表明，这类醚氧比例很少. 原因是存在负协同效应 ${ }^{[27]}$.

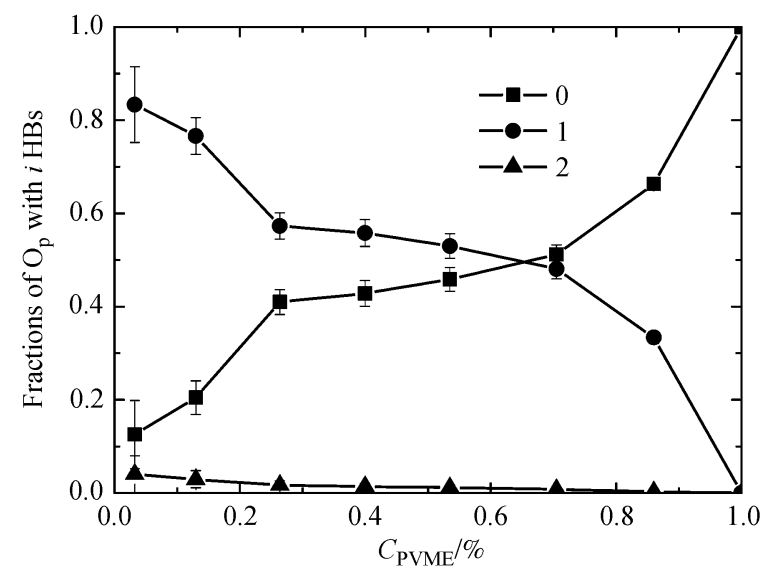

图 5 醚氧和 $i(i=0,1,2)$ 个水分子形成氢键的比例

\subsection{2 准氢键在聚合物结构单元上的分布}

前期工作 ${ }^{[5]}$ 表明准氢键对 $\mathrm{PVME} / \mathrm{H}_{2} \mathrm{O}$ 体系的物 理性质也有贡献. 图 6 给出了和 $i(i=1,2)$ 个水分子形 成准氢键的 $\mathrm{CH}_{3}$ 基团的比例. 从图中可以发现准氢键 比例随浓度的变化与图 5 所表示的氢键比例随浓度 的变化趋势一致, 转折点也大致相当. 这也是由分子 链的拓扑结构和水分子与聚合物结构单元比例减少 造成的. 形成准氢键的结构单元的比例要明显小于 形成氢键的比例. 在低浓度下, 水分子较为充足, 在 形成氢键的同时也有很多的结构单元和水分子形成 准氢键. 随着浓度的增加, 水分子的数量优势逐渐降 低，它们更加倾向于和聚合物形成单一氢键，因而形 成准氢键的结构单元比例减少. 这些结果也可以用 来解释红外谱带发生位移的主要因素 ${ }^{[5]}$. 在较低浓度 下氢键和准氢键协同作用, 而在高浓度区起主导作 用的则是醚氧和水之间的氢键作用.

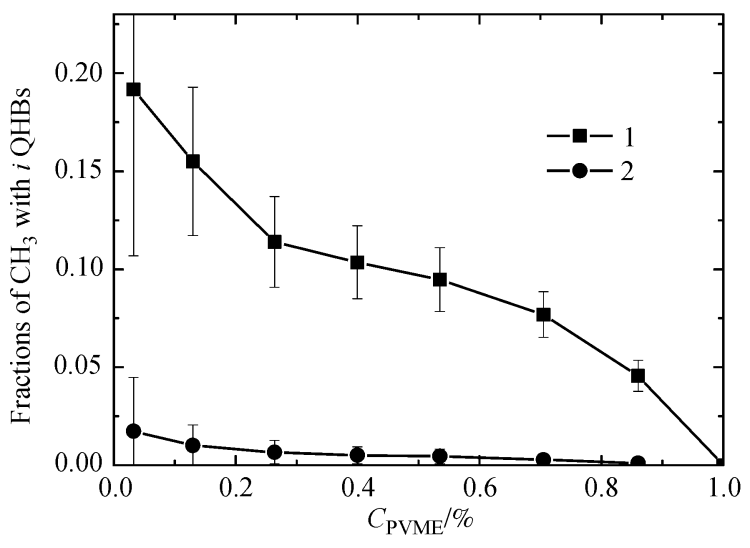

图 $6 \mathrm{CH}_{3}$ 和 $i(i=1,2)$ 个水分子形成准氢键的比例

\subsection{3 聚合物结构单元和水分子的团簇缔合}

PVME的溶剂化结构并不是单独的氢键和准氢 键就能准确描述的, 实验和理论计算 ${ }^{[5,10]}$ 表明PVME 的结构单元在水溶液中可以和 5 个以内的水分子形 成团簇结构. 氢键和准氢键往往相互“协作”而在聚合 物周围形成不同的聚集体 ${ }^{[5,10,33 ~ 35]}$, 另外形成聚集体 的水分子之间的氢键也对宏观性质有着不可或缺的 贡献。

如果在没有氢键缔合的结构单元上形成的准氢 键是不能够稳定存在的 ${ }^{[5]}$, 通过图 5 和图 6 可以求出 每个形成氢键的结构单元平均缔合氢键和准氢键的 数目. 发生氢键缔合的醚氧其缔合数量在各浓度变 化不大，均在 1.0 附近，这也反映了负协同效应 ${ }^{[27]}$ 的 影响。在和水分子发生缔合的结构单元当中有 $20 \%$ 左右的 $\mathrm{CH}_{3}$ 基团和水分子形成准氢键.

为了获得聚合物结构单元和水分子缔合更为清 晰的物理图像，我们假定在 $86 \%$ 时溶液中已经没有 “自由水”，即所有的水分子都通过氢键(包括不直接 和醚氧相连的)或者准氢键和聚合物发生缔合. 那么 在和水分子发生缔合的结构单元当中，每个结构单 元大约与 1.56 个水分子缔合. 该值与前期工作 ${ }^{[5]}$ 得到 的缔合数 (1.5)相当. 在质量分数分别为 $70 \% 、 54 \%$ 和 $40 \%$ 下，每个和水分子发生缔合的结构单元可以分别 分配到 2.77、5.18 和 8.48 个水分子，由此可以推断当 浓度在 $54 \%$ 以下，溶液中出现了较多的“自由水”. 从 文献 [25]中的相图可以发现，PVME/ $\mathrm{H}_{2} \mathrm{O}$ 体系的 
LCST曲线一个最低点在 $10 \%$ 附近，另一个在 $65 \%$ 附 近，上文已经证明在这两个浓度附近体系的结构的 差别：在较低浓度，体系中存在大量“自由水”, 大多 数结构单元和水分子形成氢键或准氢键; 在较高浓 度, 体系中大部分水分子和聚合物直接缔合成氢键. 这种结构和微观作用上的差异一直被认为是“W”形 LCST曲线产生的主要原因 ${ }^{[10,34]}$.

\section{4 结论}

本文利用分子动力学方法模拟了 $300 \mathrm{~K}$ 下不同 浓度聚甲基乙烯基醚(PVME)水溶液的微观溶剂化结 构. RDF 和氢键 DA 距离的分布都表明 $\mathrm{O}_{\mathrm{p}}-\mathrm{O}_{\mathrm{w}}$ 氢键 比 $\mathrm{O}_{\mathrm{w}}-\mathrm{O}_{\mathrm{w}}$ 氢键短 $0.005 \mathrm{~nm}$ 左右. $\mathrm{C}-\mathrm{H} \cdots \mathrm{O}$ 准氢键的 数目仅占所有范德华作用对的 $7.2 \%$ 左右.

在各浓度下，水分子并不能均匀地分布在聚合 物结构单元上, 即使在很稀的溶液 $(3.3 \%)$ 中, 仍然有 $10 \%$ 左右的醚氧没有和水分子形成氢键. 这归因于聚 合物链复杂的拓扑结构, 聚合物链之间相互穿插, 导
致一些醚氧不能和水分子有效地接触而形成氢键. 准氢键随浓度的变化和氢键的变化趋势类似，但形 成准氢键的结构单元与形成氢键的结构单元的比值 仅约 0.2. 实验上用动态 DSC 测量低分子量 PVME 水 溶液的相转变焓发现在浓度为 $30 \%$ 左右有一转折, 而计算得出在浓度为 $27 \%$ 左右氢键和准氢键比例有 一转折, 这给相转变焓的转折点提供了分子尺度的 微观解释.

在浓度分别为 $86 \%$ 和 $70 \%$ 下，每个结构单元大 约与 1.56 和 2.77 个水分子缔合. 在浓度大于 $54 \%$ 的 溶液中出现了较多的“自由水”。因此在较低浓度，体 系中存在大量“自由水”, 大多数结构单元和水分子形 成氢键或准氢键；而在高浓度区，体系中大量的水分 子和聚合物缔合成氢键.

后期工作将对 $\mathrm{PVME} / \mathrm{H}_{2} \mathrm{O}$ 体系的团簇结构及氢 键和准氢键的协同作用作进一步的探讨, 并将分析 浓度、温度和静电场对聚合物构象及溶剂化结构的影 响.

\section{参考文献}

1 Sun T, King H E. Discovery and modeling of upper critical solution behavior in the poly(ethylene ox-ide)/water system at elevated pressure. Macromolecules, 1998, 31(18): 6383-6386[DOI]

2 Tamai Y, Tanaka H, Nakanishi K. Molecular dynamics study of polymer-water interaction in hydrogels. 1. Hydrogen-bond structure. Macromolecules, 1996, 29(21): 6750-6760[DOI]

3 Nies E, Li T, Berghmans T H, Heenan R K, King S M. Upper critical solution temperature phase behavior, Composition fluctuations, and complex formation in poly (Vinyl Methyl Ether)/D2O solutions: Small-angle neutron-scattering experiments and wertheim lattice thermodynamic perturbation theory predictions. J Phys Chem B, 2006, 110(11): 5321 - 5329 [DOI]

4 van Durme K, van Assche G, Nies E, van Mele B. Phase transformations in aqueous low molar mass poly(vinyl methyl ether) solutions: Theoretical prediction and experimental validation of the peculiar solvent melting line, bimodal LCST and (Adjacent) UCST miscibility gaps. J Phys Chem B, 2007, 111(6): 1288-1295[DOI]

5 Zeng X G, Yang X Z. A study of interaction of water and model compound of poly(vinyl methyl ether). J Phys Chem B, 2004, 108(45): 17384-17392[DOI]

6 Liu Z, Calvert P. Multilayer hydrogels as muscle-like actuators. Adv Mater, 2000, 12(4): 288 - 291 [DOI]

7 Qiu Y, Park K. Environment-sensitive hydrogels for drug delivery. Adv Drug Del Rev, 2001, 53(3): 321—339[DOI]

8 Miyataa T, Uragamia T, Nakamaeb K. Biomolecule-sensitive hydrogels. Adv Drug Del Rev, 2002. 54(1): 79-98[DOI]

9 Schafer-Soenen H, Moerkerke R, Berghmans H, Koningsveld H, Dusek K, Solc K. Zero and off-zero critical concentrations in systems containing polydisperse polymers with very high molar masses. 2. The system water-poly(vinyl methyl ether). Macromolecules, 1997, 30(3): 410-416[DOI]

10 Maeda H. Interaction of water with poly(vinyl methyl ether) in aqueous solution. J Polym Sci Part B-Polym Phys, 1994, 32(1): 91—97 $\underline{\text { [DOI] }}$

11 Némethy G, Scheraga H A. Structure of water and hydrophobic bonding in proteins. II . Model for the thermodynamic properties of aqueous solutions of hydrocarbons. J Chem Phys, 1962, 36(12): 3401-3417[DOI]

12 Frank H S, Evans M W. Free volume and entropy in condensed systems III. Entropy in binary liquid mix-tures; partial molal entropy 
in dilute solutions; structure and thermodynamics in aqueous electrolytes. J Chem Phys, 1945, 13(11): 507-532[DOI]

13 Spoel D, Lindahl E, Hess B, Groenhof G, Mark A E, Berendsen H J C. Gromacs: Fast, flexible, and free. J Comput Chem, 2005, 26(16): 1701-1718[DOI]

14 Hoover W G. Canonical dynamics: Equilibrium phase-space distributions. Phys Rev A, 1985, 31(3): 1695-1697[DOI]

15 Nosé S. A molecular dynamics method for simulations in the canonical ensemble. Mol Phys, 1984, 52(2): 255-268[DOI]

16 Parrinello M, Rahman A. Polymorphic transitions in single crystals: A new molecular dynamics method. J Appl Phys, 1981, 52(12): $7182-7190[\mathrm{DOI}$

17 Essmann U, Perera L, Berkowitz M L, Darden T, Lee H, Pedersen L G. A smooth particle mesh Ewald method. J Chem Phys, 1995, 103(19): 8577-8593[DOI]

18 Darden T, York D, Pedersen L. Particle mesh Ewald: An $N \cdot \log (N)$ method for Ewald sums in large systems. J Chem Phys, 1993, 98(12): 10089-10092[DOI]

19 Hess B, Bekker H, Berendsen H J C, Fraaije J G E M. Lincs: A linear constraint solver for molecular simulations. J Comput Chem, 1997, 18(12): 1463-1472[DOI]

20 Miyamoto S, Kollman P A. Settle: An analytical version of the SHAKE and RATTLE algorithm for rigid water models. J Comput Chem, 1992, 13(8): 952-962[DOI]

21 Jorgensen W L, Chandrasekhar J, Madura J D, Impey R W, Klein M L. Comparison of simple potential functions for simulating liquid water. J Chem Phys, 1983, 79: 926-935[DOI]

22 Lawrence C P, Skinner J L. Flexible TIP4P model for molecular dynamics simulation of liquid water. Chem Phys Lett, 2003, 372: $842-847 \underline{[\mathrm{DOI}]}$

23 Kaminski G A, Friesner R A, Tirado-Rives J, Jorgensen W L. Evaluation and reparametrization of the OPLS-AA force field for proteins via comparison with accurate quantum chemical calculations on peptides. J Phys Chem B, 2001, 105: 6474-6487[DOI]

24 Jorgensen W L, Maxwell D S, Tirado-Rives J. Development and testing of the OPLS all-atom force field on conformational energetics and properties of organic liquids. J Am Chem Soc, 1996, 118: 11225-11236[DOI]

25 Loozen E, Nies E, Heremans K, Berghmans H. The influence of pressure on the lower critical solution temperature miscibility behavior of aqueous solutions of poly(vinyl methyl ether) and the relation to the compositional curvature of the volume of mixing. J Phys Chem B, 2006, 110(15): 7793-7802[DOI]

26 Shiomi T, Hamada F, Nasako T, Yoneda K, Imai K, Nakajima A. Thermodynamics of polymer blends of poly(vinyl methyl ether) and polystyrene. Macromolecules, 1990, 23(1): 229-233[DOI]

27 Scheiner S. Hydrogen Bonding. New York: Oxford University Press, 1997. 230-231

28 Luzar A, Chandler D. Structure and hydrogen bond dynamics of water-dimethyl sulfoxide mixtures by computer simulations. J Chem Phys, 1993, 98(10): 8160-8173[DOI]

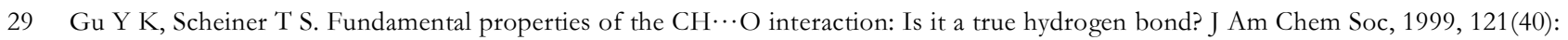
$9411-9422 \underline{\text { DOI] }}$

30 Lee H S, Wang Y K, Hsu S L. Spectroscopic analysis of phase separation behavior of model polyurethanes. Macromolecules, 1987, 20(9): 2089-2095[DOD]

31 Zhang R, Li H, Lei Y, Han S. All-atom molecular dynamic simulations and relative NMR spectra study of weak C-H $\cdots \mathrm{O}$ contacts in amide-water systems. J Phys Chem B, 2005, 109(15): 7482-7487[DOI]

32 Vargas R, Garza J, Dixon D A, Hay B P. How strong is the Ca-H $\cdots \mathrm{O}=\mathrm{C}$ hydrogen bond? J Am Chem Soc, 2000, 122(19): 47504755 [DOI]

33 Maeda Y. IR spectroscopic study on the hydration and the phase transition of poly(vinyl methyl ether) in water. Langmuir, 2001, 17(5): $1737-1742 \underline{[\mathrm{DOI}]}$

34 Meeussen F, Bauwens Y, Moerkerke R, Nies E, Berghmans H. Molecular complex formation in the system poly(vinyl methyl ether)/water. Polymer, 2000, 41(10): 3737-3743[DOI]

35 Zhang J, Berge B, Meeussen F, Nies E, Berghmans H, Shen D. Influence of the interactions in aqueous mixtures of poly(vinyl methyl ether) on the crystallization behavior of water. Macromolecules, 2003, 36(24): 9145-9153[DOI] 